\title{
CONTROL OF A CLAMPED-FREE BEAM BY A PIEZOELECTRIC ACTUATOR
}

\author{
Emmanuelle Crépeau ${ }^{1}$ and Christophe Prieur ${ }^{2}$
}

\begin{abstract}
We consider a controllability problem for a beam, clamped at one boundary and free at the other boundary, with an attached piezoelectric actuator. By Hilbert Uniqueness Method (HUM) and new results on diophantine approximations, we prove that the space of exactly initial controllable data depends on the location of the actuator. We also illustrate these results with numerical simulations.
\end{abstract}

Mathematics Subject Classification. 93C20, 35B75, 35B60.

Received September 27, 2004. Revised May 23, 2005.

\section{INTRODUCTION}

There exists now a large literature concerning the study of flexible structures in interaction with piezoelectric actuators. Two directions of research can be exhibited. The modeling problem and the controllability problem.

A large number of models have been proposed and many dynamical systems modelizing a flexible structure with a piezoelectric actuator can be found in the literature; e.g. model based on finite elements (see $[2,12]$ ), finite-dimensional approximations (see $[11,21,22]$ ) and also partial differential equations (see $[6,7]$ ).

Concerning controllability results, there exists a wide literature. See e.g. [18] where the eigenvalue specification problem is studied for the Euler-Bernoulli beam (with the same boundary condition but with different control than in this paper). See also $[8,20]$ where flatness technics are used for approximate controllability problems.

To the best to our knowledge, the only exact controllability result for a beam with a piezoelectric controller is due to [23], where a beam with hinged boundary conditions is considered. The main purpose of our paper is to study the exact controllability of a beam in a more physical configuration: the clamped-free boundary conditions, i.e. a beam clamped at one end and free at the other end. There exist technological and industrial (see [15]) motivations to study the control of beams with piezoelectric actuators with such boundary conditions.

In this paper we use the Hilbert Uniqueness Method (HUM) which is a classical approach to look for a controllability result (see [16]) combined with new results of the theory of diophantine approximations.

With such physical conditions, the controllability problem is a completely different issue from [23] although the method used in this paper and in [23] is the same one (namely HUM). Indeed the computations and the diophantine approximations used in [23] and in this paper are slightly different and here we use some new results in the theory of diophantine approximations.

\footnotetext{
Keywords and phrases. piezoelectric actuator, metallic beam, exact controlability.

1 Bâtiment Fermat, Université de Versailles Saint-Quentin, 45 avenue des États-Unis, 78035 Versailles, France;

crepeau@math.uvsq.fr

${ }^{2}$ LAAS - CNRS, 7 avenue du Colonel Roche, 31077 Toulouse, France; Christophe.Prieur@laas.fr
} 
We prove that for any time $T>0$ we have exact controllability of the beam if the position of patch is chosen correctly. Roughly speaking, this exact controllability property means that the solutions of our model is at the equilibrium at time $T$ for sufficiently regular initial conditions.

Three main results are proved in this paper (see Sect. 1 for a precise statement):

- For almost every position of the piezoelectric patch's ends, all initial conditions of the beam in a sufficiently regular space are exactly controllable at time $T>0$, for every $T>0$.

- There exists a zero Lebesgue measure set of the piezoelectric patch's ends, such that all initial conditions of the beam in a less regular space are exactly controllable at time $T$, for every $T>0$.

- If the length of the patch is exactly the length of the beam, then we have exact controllability results in a larger class of initial conditions than the class of controllable initial conditions with the boundary condition of [23].

Moreover we compute explicitly the voltage on the actuator, we need to apply to get these exact-controllability properties. We check on numerical simulations that the exact-controllability is obtained. To do this we consider the physical scaling parameters and look for the applicability of our control law.

The paper is organized as follows. In Section 1, we introduce the model of the beam under consideration and give the main results of the paper. In Section 2, we give some preliminary results and then we state the existence and give some regularity results of the Cauchy problem in Section 3. The main results are proved in Section 4, and in Section 5, numerical simulations and physical applicability are studied.

Finally in Appendix A we prove the diophantine approximations needed for the controllability results of Section 4.

\section{Modelization AND MAIN RESUlts}

Let us consider a Bernoulli-Euler beam that is free at one end and clamped at the opposite end. The beam is subject to an attached piezoelectric actuator. Our model under consideration is the following one (see $[9,10,17]$ e.g.), for every $(x, t)$ in $[0, L] \times[0, T]$,

$$
\begin{array}{r}
w_{t t}(x, t)+\frac{Y I}{\rho A_{b}} w_{x x x x}(x, t)=\frac{1}{\rho A_{b}} m_{x x}(x, t), \\
w(0, t)=0, w_{x}(0, t)=0, \\
w_{x x}(L, t)=0, w_{x x x}(L, t)=0,
\end{array}
$$

where

- $x \in[0, L]$ denotes the spatial coordinate attached to the beam of length $L$;

- $t \in[0, T]$ is the time coordinate, $T>0$;

- $x=0$ is the clamped boundary of the beam;

- $x=L$ is the free boundary of the beam;

- $w(x, t)$ is the beam transverse deflection at point $x$ and at time $t$;

- $\rho$ is the (uniform) density of the beam;

- $A_{b}$ is the cross-sectional area;

- $Y$ is the Young's modulus of elasticity;

- $I$ is the moment of inertia of the beam;

- $m$ is the moment acting on the beam due to the piezoelectric actuator.

The initial conditions are

$$
w(., 0)=w^{0}, w_{t}(., 0)=w^{1} .
$$

The piezoelectric actuator acts a moment on the beam due to the applied voltage. This moment is only located on the actuator. It is expressed by (see $[10,15])$ :

$$
m(x, t)=K_{a} V_{a}(t)[H(x-\xi)-H(x-\eta)],
$$


where $K_{a}$ is a constant based on the properties of the beam and the piezoceramic patches (see an expression in Sect. 5.1), $V_{a}(t)$ is the applied voltage to the piezoelectric actuator and $x \mapsto H(x)$ is the Heaviside's mapping, i.e. vanishing for every $x<0$, and equal to 1 for every $x \geq 0$. Here $\xi$ and $\eta$ denote the location of the piezoelectric patch's ends along the $x$-axis.

To state our main results, let us introduce the function space $Y_{\alpha}$ defined as follows, for any $\alpha \in \mathbb{R}$. Let $Y_{0}=\mathrm{L}^{2}(0, L)$. For every $\alpha>0$, let $Y_{\alpha}$ be the closure in $H^{\alpha}(0, L)$ of the $y \in \mathrm{C}^{\infty}([0, L])$ satisfying, for every $n$ in $\mathbb{N}$,

$$
\begin{gathered}
y^{(4 n)}(0)=0, \\
y^{(4 n+1)}(0)=0, \\
y^{(4 n+2)}(L)=0, \\
y^{(4 n+3)}(L)=0
\end{gathered}
$$

For every $\alpha>0$, we define $Y_{-\alpha}$ as the dual space of $Y_{\alpha}$ with respect to the space $Y_{0}$.

Our exact controllability results are

Theorem 1.1. Let $\epsilon>0$, there exists a set $A$ in $(0, L)^{2}$ with Lebesgue measure $L^{2}$ such that, for every $(\xi, \eta) \in A$, all initial conditions in $Y_{3+\epsilon} \times Y_{1+\epsilon}$ are exactly $\mathrm{L}^{2}(0, T)$-controllable at time $T>0$, for every $T>0$.

Theorem 1.2. There exists a non-empty set $B$ (uncountable but with Lebesgue measure zero) in $(0, L)^{2}$ such that, for every $(\xi, \eta) \in B$, all initial conditions in $Y_{3} \times Y_{1}$ are exactly $\mathrm{L}^{2}(0, T)$-controllable at time $T>0$, for every $T>0$.

Theorem 1.3. In the special case of $(\xi, \eta)=(0, L)$, all initial conditions in $Y_{1} \times Y_{-1}$ are exactly $\mathrm{L}^{2}(0, T)$ controllable at time $T>0$, for every $T>0$.

Let us prove some preliminary results which are useful for the proof of our main results.

\section{Preliminaries}

By rescaling time and space, we may assume in all the following (except in Sect. 5) that $\frac{Y I}{\rho A_{b}}=1$ and $L=\pi$. Let us define $u(t)=\frac{1}{\rho A_{b}} K_{a} V_{a}(t)$ and thus, the controlled problem under consideration becomes

$$
\begin{gathered}
w_{t t}+w_{x x x x}=u(t)(H(x-\xi)-H(x-\eta))_{x x}, 0<x<\pi, 0<t<T \\
w(0, t)=0, w_{x}(0, t)=0 \\
w_{x x}(\pi, t)=0, w_{x x x}(\pi, t)=0 \\
w(., 0)=w^{0}, w_{t}(., 0)=w^{1} .
\end{gathered}
$$

Since the problem is time-reversible we only need to considerate a null-controllability issue. That is, we say that the initial conditions $w^{0}, w^{1}$ are exactly controllable at time $T$ if there exists $u \in \mathrm{L}^{2}(0, T)$ such that the solution $w$ of $(6)-(9)$ satisfies

$$
w(., T)=0, w_{t}(., T)=0 .
$$

Let us introduce the homogeneous Cauchy problem:

$$
\begin{gathered}
\phi_{t t}+\phi_{x x x x}=0, \\
\phi(0, t)=0, \phi_{x}(0, t)=0, \\
\phi_{x x}(\pi, t)=0, \phi_{x x x}(\pi, t)=0, \\
\phi(., 0)=\phi^{0}, \phi_{t}(., 0)=\phi^{1} .
\end{gathered}
$$


Let us end this section by studying the eigenfunctions of the operator $w \mapsto w_{x x x x}$ and giving some asymptotic behaviors:

Lemma 2.1. Let $\mathcal{A}: H^{4}(0, \pi) \rightarrow \mathrm{L}^{2}(0, \pi)$ be defined, for every $w \in H^{4}(0, \pi)$, by

$$
\mathcal{A} w=w_{x x x x},
$$

with

$$
\mathcal{D}(\mathcal{A})=\left\{w \in H^{4}(0, \pi), w(0)=w_{x}(0)=w_{x x}(\pi)=w_{x x x}(\pi)=0\right\} .
$$

Then the $\mathrm{L}^{2}(0, \pi)$-normalized eigenfunctions of $\mathcal{A}$ are, for every $k$ in $\mathbb{N}^{*}$,

$$
\psi_{k}(x)=\gamma_{k}\left(\cos \left(\alpha_{k} x\right)-\cosh \left(\alpha_{k} x\right)+\mu_{k}\left(\sinh \left(\alpha_{k} x\right)-\sin \left(\alpha_{k} x\right)\right)\right),
$$

where $\alpha_{k}$ is the $k$-th positive root of

$$
1+\cos \left(\alpha_{k} \pi\right) \cosh \left(\alpha_{k} \pi\right)=0
$$

and

$$
\begin{aligned}
\mu_{k} & =\frac{\cos \left(\alpha_{k} \pi\right)+\cosh \left(\alpha_{k} \pi\right)}{\sin \left(\alpha_{k} \pi\right)+\sinh \left(\alpha_{k} \pi\right)}, \\
\gamma_{k} & =\frac{1}{\sqrt{\pi}},
\end{aligned}
$$

for the eigenvalue

$$
\lambda_{k}=\alpha_{k}^{4} \text {. }
$$

Moreover we have the following Taylor expansions, as $k$ tends to $+\infty$ :

$$
\begin{gathered}
\alpha_{k}=k-\frac{1}{2}+(-1)^{k+1} \frac{2 \mathrm{e}^{\frac{\pi}{2}}}{\pi} \mathrm{e}^{-\pi k}+o\left(\mathrm{e}^{-\pi k}\right), \\
\mu_{k}=1+2(-1)^{k} \mathrm{e}^{-\alpha_{k} \pi}+o\left(\mathrm{e}^{-\alpha_{k} \pi}\right),
\end{gathered}
$$

and also, for every $\rho \in\left(0, \frac{\pi}{2}\right)$,

$$
-\sinh \left(\alpha_{k} \rho\right)+\mu_{k} \cosh \left(\alpha_{k} \rho\right)=\mathrm{e}^{-\alpha_{k} \rho}+o\left(\mathrm{e}^{-\alpha_{k} \rho}\right),
$$

for every $\rho \in\left(\frac{\pi}{2}, \pi\right)$,

$$
-\sinh \left(\alpha_{k} \rho\right)+\mu_{k} \cosh \left(\alpha_{k} \rho\right)=(-1)^{k} \mathrm{e}^{-\alpha_{k}(\pi-\rho)}+o\left(\mathrm{e}^{-\alpha_{k}(\pi-\rho)}\right) .
$$

Proof. Thanks to (14) the eigenfunctions of $\mathcal{A}$ are a linear combination of $x \mapsto \cos \left(\alpha_{k} x\right), x \mapsto \sin \left(\alpha_{k} x\right)$, $x \mapsto \cosh \left(\alpha_{k} x\right)$ and $x \mapsto \sinh \left(\alpha_{k} x\right)$ for the eigenvalue $\lambda_{k}=\alpha_{k}^{4}$ with $\alpha_{k}$ positive. Due to the boundary conditions at $x=0$, we have (15), for $\alpha_{k}$ positive satisfying (19) and for some $\gamma_{k}$ and $\mu_{k}$ in $\mathbb{R}$. Easy computations yield:

$$
\begin{array}{r}
\left(\psi_{k}\right)_{x x}(\pi)=\gamma_{k} \alpha_{k}^{2}\left(-\cos \left(\alpha_{k} \pi\right)-\cosh \left(\alpha_{k} \pi\right)+\mu_{k}\left(\sinh \left(\alpha_{k} \pi\right)+\sin \left(\alpha_{k} \pi\right)\right)\right) \\
\left(\psi_{k}\right)_{x x x}(\pi)=\gamma_{k} \alpha_{k}^{3}\left(\sin \left(\alpha_{k} \pi\right)-\sinh \left(\alpha_{k} \pi\right)+\mu_{k}\left(\cosh \left(\alpha_{k} \pi\right)+\cos \left(\alpha_{k} \pi\right)\right)\right) .
\end{array}
$$

Moreover note that (24) implies (17), and that (25), together with (24) implies (16). Tedious computation gives $\int_{0}^{\pi}\left(\psi_{k}\right)^{2} \mathrm{~d} x=1$ with $\gamma_{k}=\frac{1}{\sqrt{\pi}}$. 
Let us now prove (20). Note that, due to the periodicity of cos, we have

$$
\alpha_{k}=k-\frac{1}{2}+\frac{\beta_{k}}{\pi}, k-1<\alpha_{k}<k \text { and } \frac{1}{2}<\alpha_{k},
$$

where $\left(\beta_{k}\right)_{k}$ is a sequence such that $\beta_{k} \rightarrow 0$ as $k$ tends to infinity. Due to (16), we have

$$
2+(-1)^{k} \sin \left(\beta_{k}\right)\left(\mathrm{e}^{\pi k-\frac{\pi}{2}+\beta_{k}}+\mathrm{e}^{-\pi k+\frac{\pi}{2}-\beta_{k}}\right)=0 .
$$

This implies

$$
2 \mathrm{e}^{-\pi k}+(-1)^{k}\left(\beta_{k}+o\left(\beta_{k}\right)\right)\left(\mathrm{e}^{-\frac{\pi}{2}}\left(1+\beta_{k}+o\left(\beta_{k}\right)\right)+\mathrm{e}^{-2 \pi k} \mathrm{e}^{\frac{\pi}{2}}\left(1-\beta_{k}+o\left(\beta_{k}\right)\right)\right)=0,
$$

i.e.

$$
2 \mathrm{e}^{-\pi k}+(-1)^{k} \beta_{k} \mathrm{e}^{-\frac{\pi}{2}}+o\left(\beta_{k}\right)=0
$$

which implies (20) with (26).

Let us now check the Taylor expansion (21).

From (17) and (20), we get

$$
\begin{aligned}
\mu_{k}-1 & =\frac{\sqrt{2} \sin \left(-\alpha_{k} \pi+\frac{\pi}{4}\right)+\mathrm{e}^{-\alpha_{k} \pi}}{\sin \left(\alpha_{k} \pi\right)+\sinh \left(\alpha_{k} \pi\right)} \\
& =\frac{(-1)^{k}+\mathrm{e}^{-\alpha_{k} \pi}+o\left(\mathrm{e}^{-\alpha_{k} \pi}\right)}{\sin \left(\alpha_{k} \pi\right)+\sinh \left(\alpha_{k} \pi\right)}
\end{aligned}
$$

and thus we have (21). Thanks to (20) and (21), easy computations yield (22). This concludes the proof of Lemma 2.1.

Note that, with the theory of compact and self-adjoint operators, we get that the eigenvectors form an orthonormal basis of $\mathrm{L}^{2}(0, \pi)$.

Let us now study the existence of solutions for the Cauchy problem and give some regularity results.

\section{Existence AND REGUlARITY OF SOLUTIONS}

We first study the Cauchy problem (10)-(13) and write down an estimation of $\left\|\phi_{x}(\rho, .)\right\|_{\mathrm{L}^{2}(0, T)}$ as follows.

Proposition 3.1. For every $\left(\phi^{0}, \phi^{1}\right) \in Y_{1} \times Y_{-1}$, the solution of the homogeneous problem (10)-(13) is written in Fourier series as

$$
\phi(x, t)=\sum_{k=1}^{+\infty}\left(\phi_{k}^{0} \cos \left(\alpha_{k}^{2} t\right)+\frac{\phi_{k}^{1}}{\alpha_{k}^{2}} \sin \left(\alpha_{k}^{2} t\right)\right) \psi_{k}(x)
$$

where

$$
\phi_{k}^{0}=\int_{0}^{L} \phi^{0}(x) \psi_{k}(x) \mathrm{d} x, \phi_{k}^{1}=\int_{0}^{L} \phi^{1}(x) \psi_{k}(x) \mathrm{d} x .
$$

Moreover, there exists $C>0$, such that for every $\left(\phi^{0}, \phi^{1}\right) \in Y_{1} \times Y_{-1}$ and for any $\rho \in(0, \pi)$, the function $\phi_{x}(\rho,$.$) is in \mathrm{L}^{2}(0, T)$ and

$$
\left\|\phi_{x}(\rho, .)\right\|_{\mathrm{L}^{2}(0, T)} \leq C\left(\left\|\phi^{0}\right\|_{Y_{1}}+\left\|\phi^{1}\right\|_{Y_{-1}}\right) .
$$

Proof. By the semigroup theory, we obtain that if $\left(\phi^{0}, \phi^{1}\right) \in Y_{1} \times Y_{-1}$ then the homogeneous problem (10)-(13) has a unique solution $\phi \in \mathrm{C}^{0}\left([0,+\infty), Y_{1}\right) \cap \mathrm{C}^{1}\left([0,+\infty), Y_{-1}\right)$ which is $(27)$. 
To prove (28), we use the following Ingham inequality, (see for example $[1,13]$ ) to our problem:

Lemma 3.2. Let $\nu_{k} \in \mathbb{R}^{\mathbb{Z}}$ be a sequence of pairwise distinct real numbers such that

$$
\lim _{|k| \rightarrow \infty}\left(\nu_{k+1}-\nu_{k}\right)=+\infty
$$

and let $T>0$. Then there exist two strictly positive constants $C_{1}$ and $C_{2}$ such that, for every sequence $\left(x_{k}\right)_{k} \in \mathrm{L}^{2}(\mathbb{Z})$, the series $f(t)=\sum_{-\infty}^{+\infty} x_{k} \mathrm{e}^{i \nu_{k} t}$ converges in $\mathrm{L}^{2}(0, T)$ and it satisfies

$$
C_{1} \sum_{-\infty}^{+\infty}\left|x_{k}\right|^{2} \leq \int_{0}^{T}\left|\sum_{-\infty}^{+\infty} x_{k} \mathrm{e}^{i \nu_{k} t}\right|^{2} \mathrm{~d} t \leq C_{2} \sum_{-\infty}^{+\infty}\left|x_{k}\right|^{2} .
$$

First of all, we have,

We apply Lemma 3.2 with

$$
\phi^{0}(x)=\sum_{k=1}^{\infty} \phi_{k}^{0} \psi_{k}(x) \text { and } \phi^{1}(x)=\sum_{k=1}^{\infty} \phi_{k}^{1} \psi_{k}(x)
$$

$$
\begin{gathered}
f(t)=\sum_{-\infty}^{+\infty} x_{k} \mathrm{e}^{i \nu_{k} t}, \\
\nu_{k}=-\nu_{-k}=\alpha_{k}^{2}, \\
2 x_{k}=2 \bar{x}_{-k}=\left(\phi_{k}^{0}-\frac{i \phi_{k}^{1}}{\alpha_{k}^{2}}\right) \psi_{k}^{\prime}(\rho),
\end{gathered}
$$

and we get, for any $T>0$, the existence of a constant $C_{3}>0$ such that, for every $\left(\phi^{0}, \phi^{1}\right) \in Y_{1} \times Y_{-1}$, we have

$$
\int_{0}^{T}\left|\phi_{x}(\rho, t)\right|^{2} \mathrm{~d} t \leq C_{3} \sum_{k=1}^{\infty}\left(\left|\phi_{k}^{0}\right|^{2}+\left|\frac{\phi_{k}^{1}}{\alpha_{k}^{2}}\right|^{2}\right)\left|\psi_{k}^{\prime}(\rho)\right|^{2} .
$$

But, with (15), we get

$$
\left.\psi_{k}^{\prime}(\rho)=\frac{\alpha_{k}}{\sqrt{\pi}}\left(-\sin \left(\alpha_{k} \rho\right)-\sinh \left(\alpha_{k} \rho\right)+\mu_{k}\left(\cosh \left(\alpha_{k} \rho\right)-\cos \left(\alpha_{k} \rho\right)\right)\right)\right) .
$$

Thus, with (22), there exists $C_{4}>0$ such that for every $k \in \mathbb{N}^{*}$

$$
\left|\psi_{k}^{\prime}(\rho)\right|^{2} \leq C_{4} \alpha_{k}^{2}
$$

With (29) and (30), we get,

$$
\begin{aligned}
\int_{0}^{T}\left|\phi_{x}(\rho, t)\right|^{2} \mathrm{~d} t & \leq C_{3} C_{4}\left(\left|\alpha_{k} \phi_{k}^{0}\right|^{2}+\left|\frac{\phi_{k}^{1}}{\alpha_{k}}\right|^{2}\right) \\
& \leq C\left(\left\|\phi^{0}\right\|_{Y_{1}}^{2}+\left\|\phi^{1}\right\|_{Y_{-1}}^{2}\right) .
\end{aligned}
$$

This concludes the proof of Proposition 3.1.

We now prove a theorem of existence and regularity for the problem (6)-(9).

Proposition 3.3. Let $u \in \mathrm{L}^{2}(0, \pi)$ and $\left(w^{0}, w^{1}\right) \in Y_{1} \times Y_{-1}$ then the problem (6)-(9) has a unique solution $w \in \mathrm{C}^{0}\left([0, T], Y_{1}\right) \cap \mathrm{C}^{1}\left([0, T], Y_{-1}\right)$. 
Proof. Let $\tau \in[0, T]$. Due to the linearity of (6) we consider the case $w^{0}=w^{1}=0$. Let $g \in \mathrm{C}_{0}^{\infty}$, in a first time and let $\phi$ be the solution of the following backward problem,

$$
\begin{gathered}
\phi_{t t}+\phi_{x x x x}=0, \\
\phi(0, t)=\phi_{x}(0, t)=0, \\
\phi_{x x}(L, t)=\phi_{x x x}(L, t)=0, \\
\phi(x, \tau)=0, \phi_{t}(x, \tau)=g(x) .
\end{gathered}
$$

By multiplying (6) by $\phi$ and integrating by parts, we get,

$$
\int_{0}^{\pi} w(x, \tau) g(x) \mathrm{d} x=-\int_{0}^{\tau} u(t)\left[\phi_{x}(\xi, t)-\phi_{x}(\eta, t)\right] \mathrm{d} t .
$$

Proposition 3.1 implies that,

$$
\left|\int_{0}^{\tau} u(t)\left[\phi_{x}(\xi, t)-\phi_{x}(\eta, t)\right] \mathrm{d} t\right| \leq C\|u\|_{\mathrm{L}^{2}(0, T)}\|g\|_{Y_{-1}} .
$$

Then $w(., \tau) \in Y_{1}$, for every $\tau \in[0, T]$. We conclude as in [23], Proposition 3.1, and get Proposition 3.3.

With this result of existence at hand, we can study the controllability results. It is done in Sections 4.1 and 4.2 .

\section{EXact CONTROLlability REsults}

Three kinds of exact controllability results are given in this section depending on the location of the piezoelectric actuator.

To state or controllability results, we use the HUM method intruduced by Lions in [16]. This method relies on the proof of the observability of the adjoint problem. By multiplying (6)-(9) by the solution $\phi \in$ $\mathrm{C}^{\infty}([0, \pi] \times[0, T])$ of

$$
\begin{gathered}
\phi_{t t}+\phi_{x x x x}=0, \\
\phi(0, t)=\phi_{x}(0, t)=0, \\
\phi_{x x}(L, t)=\phi_{x x x}(L, t)=0, \\
\phi(x, T)=0, \phi_{t}(x, T)=0,
\end{gathered}
$$

and by integrating by parts on $[0, \pi] \times[0, T]$, we get successively:

$$
\begin{aligned}
\int_{0}^{\pi} \int_{0}^{T} w_{t t} \phi \mathrm{d} x \mathrm{~d} t= & \int_{0}^{\pi} \int_{0}^{T} w \phi_{t t}-\int_{0}^{\pi} w^{1}(x) \phi(x, 0) \mathrm{d} x \\
& +\int_{0}^{\pi} w^{0}(x) \phi_{t}(x, 0) \mathrm{d} x, \\
\int_{0}^{\pi} \int_{0}^{T} w_{x x x x} \phi & =\int_{0}^{\pi} \int_{0}^{T} w \phi_{x x x x} \mathrm{~d} x \mathrm{~d} t, \\
\int_{0}^{\pi} \int_{0}^{T} u(t)(H(x-\xi)-H(x-\eta))_{x x} \phi(x, t) \mathrm{d} x \mathrm{~d} t & =-\int_{0}^{\pi} \int_{0}^{T} u(t)\left(\delta_{\xi}(x)-\delta_{\eta}(x)\right) \phi_{x}(x, t) \mathrm{d} x \mathrm{~d} t \\
& -\int_{0}^{T} u(t) \phi(\pi, t) \delta_{\eta}(\pi) \mathrm{d} t .
\end{aligned}
$$


We get

$$
\int_{0}^{\pi} w^{0}(x) \phi_{t}(x, 0)-w^{1}(x) \phi(x, 0) \mathrm{d} x=-\int_{0}^{T} u(t)\left(\phi_{x}(\xi, t)-\phi_{x}(\eta, t)\right) \mathrm{d} t-\int_{0}^{T} u(t) \phi(\pi, t) \delta_{\eta}(\pi) \mathrm{d} t .
$$

Therefore, when $\eta \neq \pi$, we need to find a lower bound of

$$
\int_{0}^{T}\left|\phi_{x}(\xi, t)-\phi_{x}(\eta, t)\right|^{2} \mathrm{~d} t
$$

in terms of the norm of $\phi$ in suitable spaces. To do this we use Ingham's inequality, Lemma 3.2 to our problem, as in [4] for Boussinesq equation. Thanks to (20), we have

$$
\alpha_{k}^{2}-\alpha_{k+1}^{2} \rightarrow+\infty
$$

Thus we apply Lemma 3.2 with

$$
\begin{gathered}
f(t)=\sum_{-\infty}^{+\infty} x_{k} \mathrm{e}^{i \nu_{k} t}, \\
\nu_{k}=-\nu_{-k}=\alpha_{k}^{2}, \\
2 x_{k}=2 \bar{x}_{-k}=\left(\phi_{k}^{0}-\frac{i \phi_{k}^{1}}{\alpha_{k}^{2}}\right)\left(\psi_{k}^{\prime}(\xi)-\psi_{k}^{\prime}(\eta)\right),
\end{gathered}
$$

and we get, for any $T>0$, the existence of two constants $C_{3}$ and $C_{4}>0$ such that, for every $\left(\phi^{0}, \phi^{1}\right) \in Y_{1} \times Y_{-1}$, we have

$$
\begin{aligned}
C_{3} \sum_{k=1}^{\infty}\left(\left|\phi_{k}^{0}\right|^{2}+\left|\frac{\phi_{k}^{1}}{\alpha_{k}^{2}}\right|^{2}\right)\left|\psi_{k}^{\prime}(\xi)-\psi_{k}^{\prime}(\eta)\right|^{2} \leq \int_{0}^{T}\left|\phi_{x}(\xi, t)-\phi_{x}(\eta, t)\right|^{2} \mathrm{~d} t \\
\quad \leq C_{4} \sum_{k=1}^{\infty}\left(\left|\phi_{k}^{0}\right|^{2}+\left|\frac{\phi_{k}^{1}}{\alpha_{k}^{2}}\right|^{2}\right)\left|\psi_{k}^{\prime}(\xi)-\psi_{k}^{\prime}(\eta)\right|^{2} .
\end{aligned}
$$

Moreover, thanks to Lemma 2.1 we have

$$
\begin{aligned}
\psi_{k}^{\prime}(\xi)-\psi_{k}^{\prime}(\eta)= & \frac{\alpha_{k}}{\sqrt{\pi}}\left[-2 \sin \left(\frac{\alpha_{k}(\xi-\eta)}{2}\right) \cos \left(\frac{\alpha_{k}(\xi+\eta)}{2}\right)+2 \mu_{k} \sin \left(\frac{\alpha_{k}(\xi+\eta)}{2}\right) \sin \left(\frac{\alpha_{k}(\xi-\eta)}{2}\right)\right. \\
& \left.-\sinh \left(\alpha_{k} \xi\right)+\sinh \left(\alpha_{k} \eta\right)+\mu_{k}\left(\cosh \left(\alpha_{k} \xi\right)-\cosh \left(\alpha_{k} \eta\right)\right)\right]
\end{aligned}
$$

The estimation of (35) will be obtained by using (36), (37), Lemma 2.1 and diophantine approximations.

The key point of the proof of Theorem 1.1 is the diophantine approximation stated in Lemma 4.1 below. To prove this lemma, we only need to know the first term of the Taylor expansion of the sequence $\left(\alpha_{k}\right)_{k}$ (due to $(20)$ ),

\section{See also Remark A.2 below.}

$$
\alpha_{k}=k+o(k) .
$$

To prove a controllability result in a larger set of initial conditions (namely Th. 1.2), we need to prove another result on diophantine approximation (Lem. 4.3) depending on the first four terms of the Taylor expansion of 
$\left(\alpha_{k}\right)$ i.e., (due to $\left.(20)\right)$,

$$
\alpha_{k}=k-\frac{1}{2}+o\left(\frac{1}{k^{2}}\right) .
$$

In this Taylor expansion the second term, namely $\left(-\frac{1}{2}\right)$, is rational and the two next terms vanish. Thanks to this Taylor expansion, we succeed to prove the diophantine approximation, needed for proving Theorem 1.2. For a different Taylor expansion such a diophantine result may be very difficult or impossible to prove. See also Remark A.5.

Actually, to prove controllability in the special case of a patch of length equal to the length of the beam, we only need (16)-(17) and (26).

In the following sections we prove Theorems 1.1, 1.2 and 1.3.

\subsection{Controllability for sufficiently regular initial conditions}

Let us now prove Theorem 1.1 and show how the set $A$ is constructed. To do this let us introduce the following notations.

Let \|\|$\cdot \|_{1}$ be the distance defined by, for every $x \in \mathbb{R}$,

$$
\left\||| x\left|\|_{1}=\min _{p \in \mathbb{Z}}\right| x-p \mid\right.
$$

and $\||| \cdot||_{2}$ the following shifted distance defined by, for every $x \in \mathbb{R}$,

$$
\||| x\|_{2}=\min _{p \in \mathbb{Z}}\left|x-p-\frac{1}{4}\right| .
$$

We have to prove the following result on diophantine approximations by using these distances.

Lemma 4.1. For any $\epsilon>0$ and $i \in\{1,2\}$, there exists $A_{i} \subset(0,1)$ having its Lebesgue measure equal to 1 such that, for every $a_{i} \in A_{i}$, there exists $c_{i}>0$ such that, for every $k$ in $\mathbb{N}^{*}$, we have

$$
\left\|\alpha_{k} a_{i}\right\|_{i} \geq \frac{c_{i}}{k^{1+\epsilon}}
$$

To prove this Lemma, we need to rewrite the proof of [3], Lemma 1, page 121, on diophantine approximations. This proof is completely independent of the controllability results. Therefore we postpone it in appendix (see Sect. A.1).

The set $A \subset(0, \pi)^{2}$ is defined by

$$
A=\left\{(\xi, \eta) \in(0, \pi)^{2}, \frac{\xi-\eta}{2 \pi} \in A_{1}, \frac{\xi+\eta}{2 \pi} \in A_{2}\right\}
$$

where $A_{1}$ and $A_{2}$ are defined in Section A.1.

Proof of Theorem 1.1. To begin with, let us prove the following lemma which is a consequence of the construction of the set $A$ :

Lemma 4.2. For every $(\xi, \eta)$ in $A$ defined by (38), there exists a constant $C_{5}>0$ such that, we have, for all $k$ in $\mathbb{N}^{\star}$,

$$
\begin{gathered}
\left|\sin \left(\frac{\alpha_{k}(\xi-\eta)}{2}\right)\right| \geq \frac{C_{5}}{\alpha_{k}{ }^{1+\epsilon}}, \\
\left|-\cos \left(\frac{\alpha_{k}(\xi+\eta)}{2}\right)+\sin \left(\frac{\alpha_{k}(\xi+\eta)}{2}\right)\right| \geq \frac{C_{5}}{\alpha_{k}{ }^{1+\epsilon}} .
\end{gathered}
$$


Proof. As $\frac{\xi-\eta}{2 \pi} \in A_{1}$, we have

$$
\left|\sin \left(\frac{\alpha_{k}(\xi-\eta)}{2}\right)\right|=\left|\sin \left(\pi\left(\frac{\alpha_{k}(\xi-\eta)}{2 \pi}-p_{1}\right)\right)\right| \geq\left|\sin \left(\frac{\pi c_{1}}{\alpha_{k}^{1+\epsilon}}\right)\right| \geq \frac{c_{1}}{\alpha_{k}^{1+\epsilon}},
$$

where $p_{1}$ is the nearest integer with respect to $\frac{\alpha_{k}(\xi-\eta)}{2 \pi}$ for the distance $\||\cdot|\|_{1}$.

Moreover as $\frac{\xi+\eta}{2 \pi} \in A_{2}$, we have

$$
\begin{aligned}
\left|-\cos \left(\frac{\alpha_{k}(\xi+\eta)}{2}\right)+\sin \left(\frac{\alpha_{k}(\xi+\eta)}{2}\right)\right| & =\sqrt{2}\left|\sin \left(\frac{\alpha_{k}(\xi+\eta)}{2}-\frac{\pi}{4}\right)\right| \\
& =\sqrt{2}\left|\sin \left(\pi\left(\frac{\alpha_{k}(\xi+\eta)}{2 \pi}-\frac{1}{4}-p_{2}\right)\right)\right|, \\
& \geq \sqrt{2}\left|\sin \left(\frac{\pi c_{2}}{\alpha_{k}^{1+\epsilon}}\right)\right| \geq \sqrt{2} \frac{c_{2}}{\alpha_{k}^{1+\epsilon}},
\end{aligned}
$$

where $p_{2}$ is the nearest integer with respect to $\frac{\alpha_{k}(\xi+\eta)}{2 \pi}$ for the distance $\||\cdot|\|_{2}$.

By letting $C_{5}=\min \left(c_{1}, \sqrt{2} c_{2}\right)$, we get (39) and (40). This achieves the proof of Lemma 4.2.

Using Lemma 4.2, (21), (22) (by letting $\rho=\xi$ and $\rho=\eta$ ), (36) and (37), there exists $C_{6}>0$ such that

$$
\int_{0}^{T}\left[\phi_{x}(\xi, t)-\phi_{x}(\eta, t)\right]^{2} \mathrm{~d} t \geq C_{6} \sum_{k=1}^{\infty} \alpha_{k}^{2}\left(\left|\phi_{k}^{0}\right|^{2}+\left|\frac{\phi_{k}^{1}}{\alpha_{k}^{2}}\right|^{2}\right) \frac{1}{\alpha_{k}^{2+2 \epsilon}} \frac{1}{\alpha_{k}^{2+2 \epsilon}} .
$$

Therefore we obtain the following observability inequality:

$$
\int_{0}^{T}\left[\phi_{x}(\xi, t)-\phi_{x}(\eta, t)\right]^{2} \mathrm{~d} t \geq C_{6} \sum_{k=1}^{\infty}\left(\left|\frac{\phi_{k}^{0}}{\alpha_{k}^{1+\epsilon}}\right|^{2}+\left|\frac{\phi_{k}^{1}}{\alpha_{k}^{3+\epsilon}}\right|^{2}\right) .
$$

This concludes the proof of Theorem 1.1.

Let us now prove an other controllability result in a larger set of initial conditions.

\subsection{Controllability result for a larger set of initial conditions}

The aim of this section is to prove Theorem 1.2, i.e. a controllability result for a larger set of initial conditions than those considered in Theorem 1.1.

We have to prove the following result on diophantine approximations:

Lemma 4.3. For any $i \in\{1,2\}$, there exists a non-empty and uncountable set $B_{i} \subset(0,1)$, with Lebesgue measure 0 , such that, for all $b_{i} \in B_{i}$, there exists $c_{i}^{\prime}>0$ such that, for every $k$ in $\mathbb{N}^{*}$, we have

$$
||\left|\alpha_{k} b_{i}\right| \|_{i} \geq \frac{c_{i}^{\prime}}{k}
$$

To prove this lemma, we use a previous result on diophantine approximation (more precisely [14], p. 21) and properties of the sets under consideration in the result given in [14], page 21. The proof of Lemma 4.3 is completely independent of the controllability results. Therefore we postpone it in appendix (see Sect. A.2).

The set $B \subset(0, \pi)^{2}$ is defined by

$$
B=\left\{(\xi, \eta) \in(0, \pi)^{2}, \frac{\xi-\eta}{2 \pi} \in B_{1}, \frac{\xi+\eta}{2 \pi} \in B_{2}\right\} .
$$

By construction, the sets $B_{1}, B_{2}$ and $B$ are uncountable but with Lebesgue measure 0 . 
Proof of Theorem 1.2. To begin with, let us prove the following lemma which is a consequence of the construction of the set $B$ :

Lemma 4.4. For every $(\xi, \eta)$ in $B$ defined by (41), there exists a constant $C_{7}>0$ such that, we have

$$
\begin{gathered}
\left|\sin \left(\frac{\alpha_{k}(\xi-\eta)}{2}\right)\right| \geq \frac{C_{7}}{\alpha_{k}} \\
\left|-\cos \left(\frac{\alpha_{k}(\xi+\eta)}{2}\right)+\sin \left(\frac{\alpha_{k}(\xi+\eta)}{2}\right)\right| \geq \frac{C_{7}}{\alpha_{k}} .
\end{gathered}
$$

Proof. Due to $\frac{\xi-\eta}{2 \pi} \in B_{1}$, we have

$$
\left|\sin \left(\frac{\alpha_{k}(\xi-\eta)}{2}\right)\right|=\left|\sin \left(\pi\left(\frac{\alpha_{k}(\xi-\eta)}{2 \pi}-p_{1}\right)\right)\right| \geq\left|\sin \left(\frac{\pi c_{1}^{\prime}}{\alpha_{k}}\right)\right| \geq \frac{c_{1}^{\prime}}{\alpha_{k}}
$$

where $p_{1}$ is the nearest integer with respect to $\frac{\alpha_{k}(\xi-\eta)}{2 \pi}$ for the distance \|\|$\cdot\|\|_{1}$.

Moreover $\frac{\xi+\eta}{2 \pi} \in B_{2}$ thus, we have

$$
\begin{aligned}
\left|-\cos \left(\frac{\alpha_{k}(\xi+\eta)}{2}\right)+\sin \left(\frac{\alpha_{k}(\xi+\eta)}{2}\right)\right| & =\sqrt{2}\left|\sin \left(\frac{\alpha_{k}(\xi+\eta)}{2}-\frac{\pi}{4}\right)\right| \\
& =\sqrt{2}\left|\sin \left(\pi\left(\frac{\alpha_{k}(\xi+\eta)}{2 \pi}-\frac{1}{4}-p_{2}\right)\right)\right| \\
& \geq \sqrt{2}\left|\sin \left(\frac{\pi c_{2}^{\prime}}{\alpha_{k}}\right)\right| \geq \sqrt{2} \frac{c_{2}^{\prime}}{\alpha_{k}}
\end{aligned}
$$

where $p_{2}$ is the nearest integer with respect to $\frac{\alpha_{k}(\xi+\eta)}{2 \pi}$ for the distance \|\|$_{\cdot}\|\|_{2}$.

By letting $C_{7}=\min \left(c_{1}^{\prime}, \sqrt{2} c_{2}^{\prime}\right)$, we have (42) and (43). This achieves the proof of Lemma 4.4.

Using Lemma 4.4, (21), (22) (by letting $\rho=\xi$ and $\rho=\eta),(36)$ and (37), there exists $C_{8}>0$ such that

$$
\int_{0}^{T}\left[\phi_{x}(\xi, t)-\phi_{x}(\eta, t)\right]^{2} \mathrm{~d} t \geq C_{8} \sum_{k=1}^{\infty} \alpha_{k}^{2}\left(\left|\phi_{k}^{0}\right|^{2}+\left|\frac{\phi_{k}^{1}}{\alpha_{k}^{2}}\right|^{2}\right) \frac{1}{\alpha_{k}^{2}} \frac{1}{\alpha_{k}^{2}} .
$$

Therefore we obtain the following observability inequality:

$$
\int_{0}^{T}\left[\phi_{x}(\xi, t)-\phi_{x}(\eta, t)\right]^{2} \mathrm{~d} t \geq C_{8} \sum_{k=1}^{\infty}\left(\left|\frac{\phi_{k}^{0}}{\alpha_{k}}\right|^{2}+\left|\frac{\phi_{k}^{1}}{\alpha_{k}^{3}}\right|^{2}\right)
$$

This concludes the proof of Theorem 1.2.

\subsection{Controllability in a special case}

In this subsection, we prove exact controllability for initial states in $Y_{1} \times Y_{-1}$, when the length of the patch is equal to the length of the beam, as stated in Theorem 1.3. Due to (34) and (37), we have to find a lower bound of

$$
\psi_{k}^{\prime}(\pi)-\psi_{k}^{\prime}(0)-\psi_{k}(\pi)=\frac{2 \alpha_{k}}{\sqrt{\pi}}\left(\frac{-\sin \left(\alpha_{k} \pi\right)}{1+\frac{\sin \left(\alpha_{k} \pi\right)}{\sinh \left(\alpha_{k} \pi\right)}}\right)-\psi_{k}(\pi) .
$$


Due to (15)-(18), (20) and (21), we have

$$
\psi_{k}(\pi)=\frac{2(-1)^{k}}{\sqrt{\pi}}+o(1)
$$

Moreover, thanks to (26), $\alpha_{k}>1 / 2$ and thus $\sinh \left(\alpha_{k} \pi\right)>\sinh (\pi / 2)>1$. Hence

$$
0<1+\frac{\sin \left(\alpha_{k} \pi\right)}{\sinh \left(\alpha_{k} \pi\right)} \leq 2
$$

Then,

$$
\frac{1}{1+\frac{\sin \left(\alpha_{k} \pi\right)}{\sinh \left(\alpha_{k} \pi\right)}} \geq \frac{1}{2}
$$

Futhermore, as $\alpha_{k}>1 / 2$, then $\cosh \left(\alpha_{k} \pi\right) \geq 2$, and with $(16),-1 / 2 \leq \cos \left(\alpha_{k} \pi\right) \leq 0$ we deduce then that $\left|\sin \left(\alpha_{k} \pi\right)\right|>\frac{\sqrt{3}}{2}$ and we obtain, with (44), (45) and (46), the following inequality,

$$
\left|\psi_{k}^{\prime}(\pi)-\psi_{k}^{\prime}(0)-\psi_{k}(\pi)\right| \geq C \alpha_{k}
$$

Therefore we obtain the following observability inequality:

$$
\left.\int_{0}^{T}\left[\phi_{x}(\pi, t)-\phi_{x}(0, t)-\phi_{(} \pi, t\right)\right]^{2} \mathrm{~d} t \geq C \sum_{k=1}^{\infty}\left(\left|\frac{\phi_{k}^{0}}{\alpha_{k}^{-1}}\right|^{2}+\left|\frac{\phi_{k}^{1}}{\alpha_{k}}\right|^{2}\right) .
$$

This proves Theorem 1.3.

Remark 4.5. We easily remark that in paper [23], in this special case, the beam is not controllable in any space of the form $Y_{2-\epsilon} \times Y_{-\epsilon}$, for $\epsilon>0$.

\section{Numerical Simulations}

In this section, we check, on numerical simulations, the exact controllability. We compute also the physical voltage we need to apply on the piezoelectric-actuator to obtain the exact controllability. Moreover we look at the physical aspect of this exact controllability. First we give the mechanical values of the beam in an usual configuration and we describe our numerical algorithm in Section 5.1. Then we give the numerical simulations in Section 5.2.

\subsection{Constants and algorithm}

In this section, we apply a spectral method for numerical experiments. For greater interest, we have used the real values of the beam and of the piezoelectric actuator given in [11]. That is,

- the beam density is $\rho=2970 \mathrm{~kg} \mathrm{~m}^{-3}$;

- the beam thickness is $h=1.58 \times 10^{-3} \mathrm{~m}$, the beam width is $l=1.2 \times 10^{-2} \mathrm{~m}$ and the beam length is $L=30 \times 10^{-2} \mathrm{~m}$

- the piezoelectric thickness is $t_{a}=7.4 \times 10^{-4} \mathrm{~m}$ and the constant of charge is $d_{a 31}=-2.1 \times 10^{-10} \mathrm{~m} . \mathrm{V}^{-1}$,

- the beam Young modulus is $Y=7.8 \times 10^{10} \mathrm{~Pa}$ and the piezoelectric Young modulus is $Y_{a}=6.7 \times 10^{10} \mathrm{~Pa}$;

- the piezoelectric left position is $\xi=0 \mathrm{~m}$ and the piezoelectric right position is $\eta=3 \times 10^{-2} \mathrm{~m}$.

Moreover, we have,

$$
\begin{aligned}
& A_{b}=h l, \\
& I=\frac{l h^{3}}{12},
\end{aligned}
$$


and

$$
K_{a}=\frac{1}{2} \frac{\beta \xi(\xi+1)}{1+2 \beta \xi\left(3+6 \xi+4 \xi^{2}\right)} d_{a 31} l h^{2} Y \frac{1}{t_{a}}
$$

where $\beta=\frac{Y_{a}}{Y}$ and $\xi=\frac{t_{a}}{h}$.

We want to study numerically the problem (1)-(5).

In order to solve this problem numerically, we apply the Hilbert Uniqueness Method we have used in the previous sections, as an algorithm, see for example $[5,19]$. For sufficiently regular initial conditions, the set of positions of the patch's ends is dense in $(0, L)$.

Thus we look at the linear application, $\Lambda:\left(\phi^{0}, \phi^{1}\right) \in Y_{-1-\epsilon} \times Y_{-3-\epsilon} \mapsto\left(w_{t}(., 0), w(., 0)\right) \in Y_{1+\epsilon} \times Y_{3+\epsilon}$ defined as follows:

Let $\phi$ be the solution of the following homogeneous problem,

$$
(I)\left\{\begin{array}{l}
\phi_{t t}+\frac{Y I}{\rho A_{b}} \phi_{x x x x}=0 \\
\phi(0, .)=0, \phi_{x}(0, .)=0 \\
\phi_{x x}(L, .)=0, \phi_{x x x}(L, .)=0 \\
\phi(., 0)=\phi_{0}, \phi_{t}(., 0)=\phi_{1}
\end{array}\right.
$$

We then consider $w$ solution of the backward problem,

$$
(I I)\left\{\begin{array}{l}
w_{t t}+\frac{Y I}{\rho A_{b}} w_{x x x x}=\frac{1}{\rho A_{b}} K_{a} V_{a}(t)\left[H_{x x}(x-\xi)-H_{x x}(x-\eta)\right] \\
w(0, .)=0, w_{x}(0, .)=0 \\
w_{x x}(L, .)=0, w_{x x x}(L, .)=0 \\
w(., T)=0, w_{t}(., T)=0
\end{array}\right.
$$

with

$$
V_{a}(t)=\phi_{x}(\xi, t)-\phi_{x}(\eta, t)
$$

Thanks to Theorem 1.1, the application $\Lambda$ is invertible (except for a Lebesgue measure zero of $(\xi, \eta)$ ). We approximate this linear application with a matrix computed on a truncated basis of the eigenfunctions defined in $(15)$.

We then apply the following algorithm.

Let $T>0$ and $N$ a positive integer, let $w^{0}, w^{1}$ some initial conditions in $Y_{3+\epsilon} \times Y_{1+\epsilon}$.

(1) We look for the matrix $\Lambda_{N} \in \mathcal{M}_{2 N}$ associated with $\Lambda$ in the finite space generated by $\left(\psi_{k}\right)_{k=1, \ldots, N}$.

(a) For $k=1, \ldots, N$, we solve $(I)$ with the initial conditions $\phi^{0}(x)=\psi_{k}(x)$ and $\phi^{1}(x)=0$. We then solve $(I I)$ and get $\Lambda(., k)$.

(b) For $k=1, \ldots, N$, we solve $(I)$ with the initial conditions $\phi^{0}(x)=0$ and $\phi^{1}(x)=\psi_{k}(x)$. We then solve $(I I)$ and get $\Lambda(., k+N)$.

(2) Thus, let $\left(\phi^{0}, \phi^{1}\right)=\Lambda_{N}^{-1}\left(w^{1}, w^{0}\right)$, we solve (I) with these initial conditions and get the desired control $V_{a}$ by (47).

We implement this algorithm on Matlab to obtain we following figures. These programs can be sent upon request from the authors.

\subsection{Numerical results}

In the following simulations, we have taken $T=1, N=20$. For the first case, we have taken as initial conditions,

$$
w(x, 0)=\frac{1}{3}\left(0.01 \psi_{1}(x)+0.05 \psi_{2}(x)\right), w_{t}(x, 0)=\frac{0.01}{3} \psi_{1}(x)
$$

Figure 1 shows that the computed control allows the profile of the beam to be null at $T$. Thus, this algorithm based on Hilbert Uniqueness Method can be used to calculate each control for every initial conditions. See also 

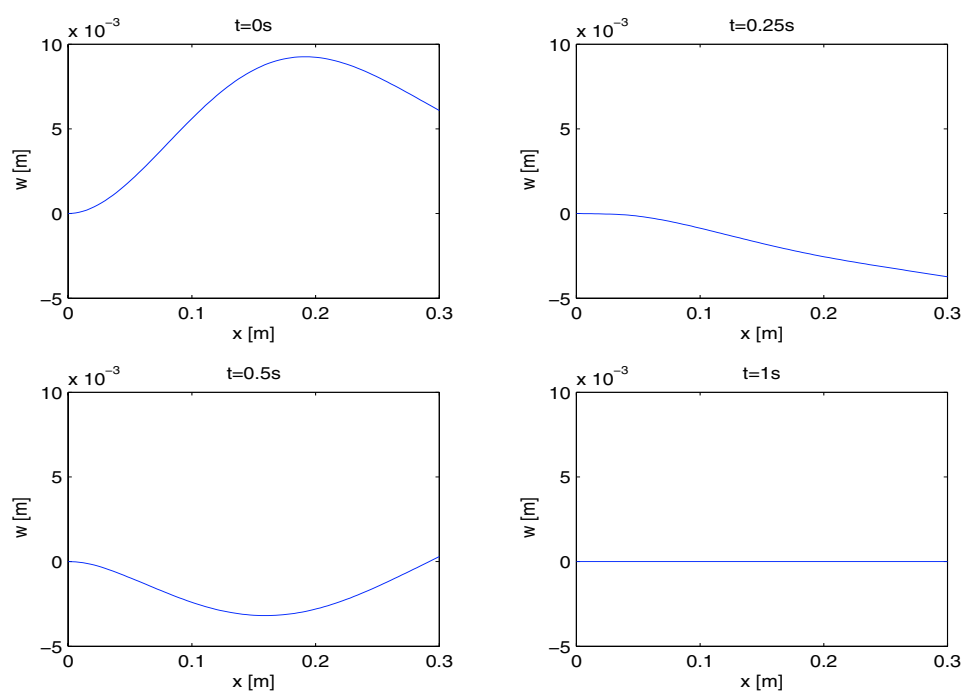

Figure 1. Profile of the beam at $t=0, t=0.25, t=0.5$ and $t=1 \mathrm{~s}$ for $T=1 \mathrm{~s}$.

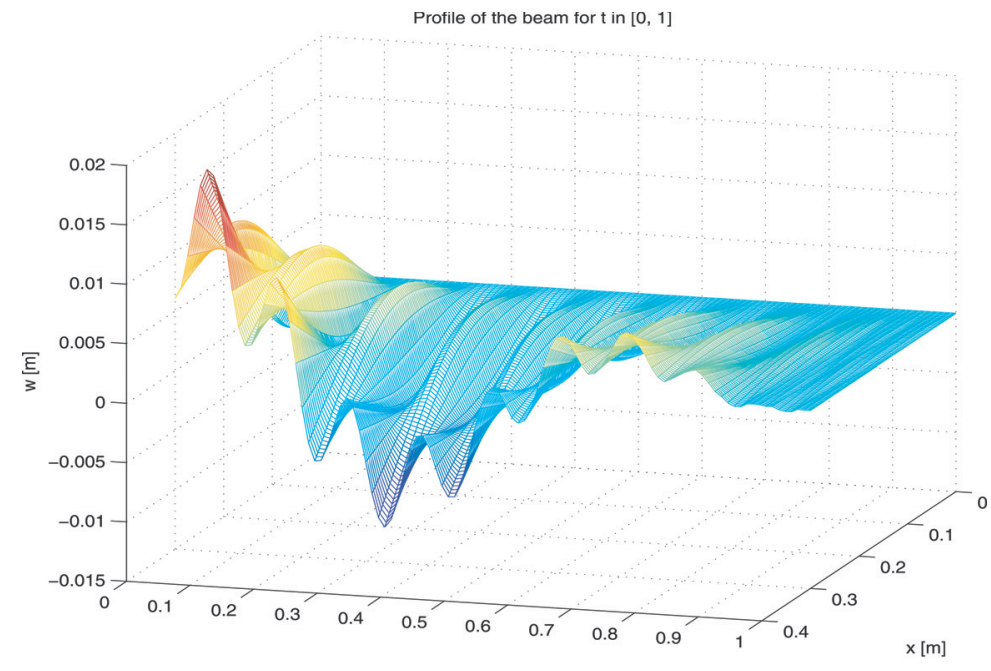

$t[s]$

Figure 2. Profile of the beam at $t \in[0, T]$ for $T=1 \mathrm{~s}$.

Figure 2. The computed voltage we need to apply to obtain such an exact controllability result is drawn at Figure 3. Note that the voltage applied on the piezoelectric actuator is in $[-5000 \mathrm{~V}, 5000 \mathrm{~V}]$.

But, if we have in mind to do such real experiences, we have some constraints on the voltage we can apply on the piezoactuator, as $V \in[-400 \mathrm{~V}, 400 \mathrm{~V}]$ (see [15]). Hence, with the initial deformation (48), we have to take a longer time of control. For the control time $T=12$, the profile of the beam at different time instants is given by Figure 4 and the voltage we need to apply is drawn at Figure 5 . We check that this voltage is between $-400 \mathrm{~V}$ and $400 \mathrm{~V}$.

We have plotted in Figure 6, the $L^{2}$-norm of the control as a function of the final time $T$. Since the control given by HUM gives the optimal control with respect to the quadratic cost $\int_{0}^{T} V^{2}(t) \mathrm{d} t$ (see [16]), we expect that this function is nonincreasing. This remark is validated with Figure 6. 


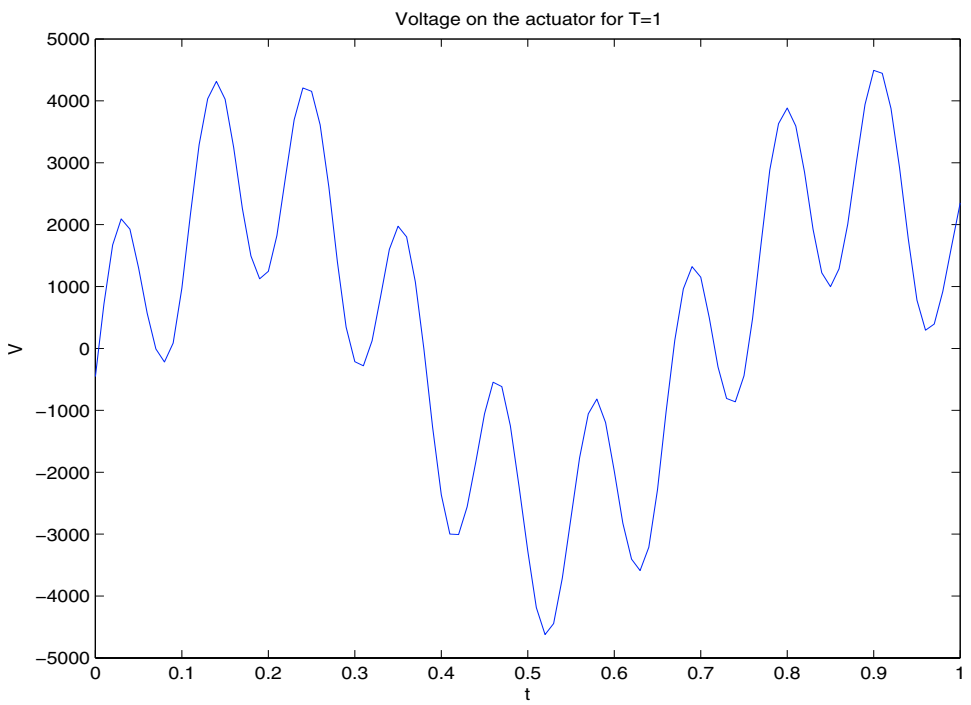

FigURE 3. Voltage applied to the piezoelectric actuator for $T=1 \mathrm{~s}$.
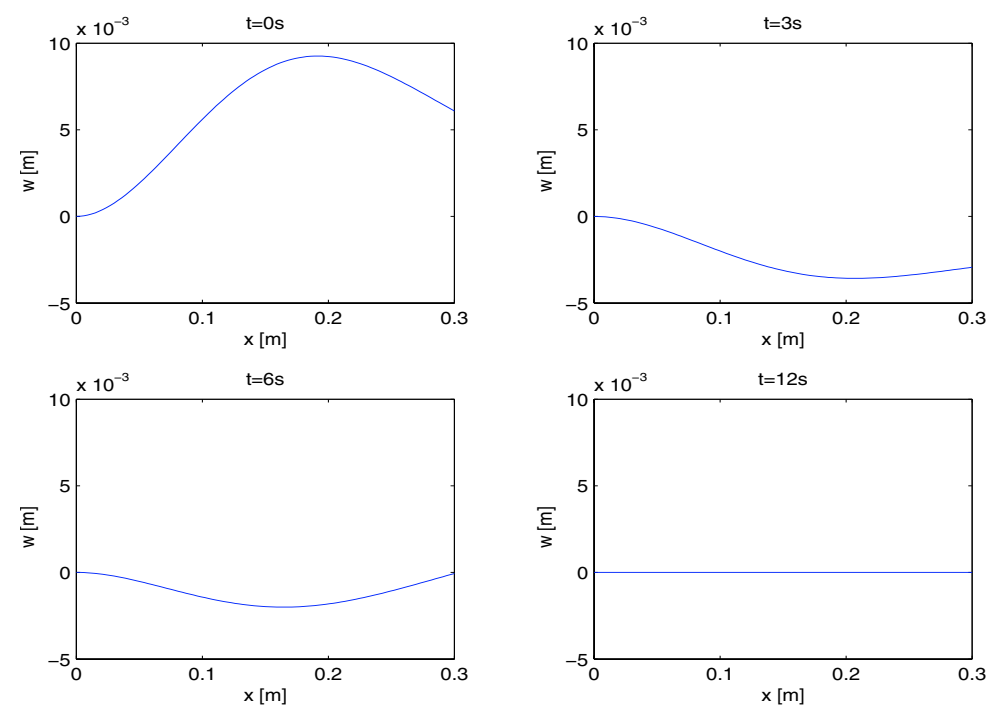

Figure 4. Profile of the beam at $t \in[0, T]$ for $T=12 \mathrm{~s}$.

\section{Conclusion}

In this paper we get some results that are similar in one way to the results obtained by Tucsnak in [23], namely Theorems 1.1 and 1.2. To prove these theorems, we need to prove new results in the theory of diophantine approximations. We can notice that Theorem 1.3 is very different. Indeed, Tucsnack has proved that there is no controllability in spaces like $Y_{\epsilon+2} \times Y_{\epsilon}$ for any $\epsilon>-2$ and we got controllability results in $Y_{1} \times Y_{-1}$. We did not manage to prove a result like Tucsnak's result [23] for the other positions of the patch. 


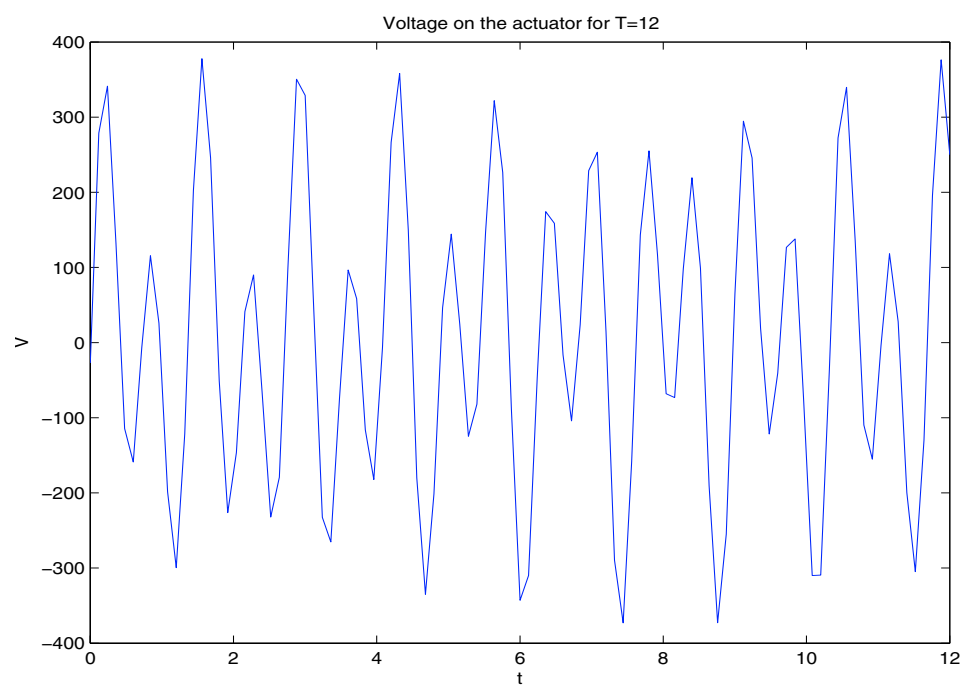

FiguRE 5. Voltage applied to the piezoelectric actuator for $T=12 \mathrm{~s}$.

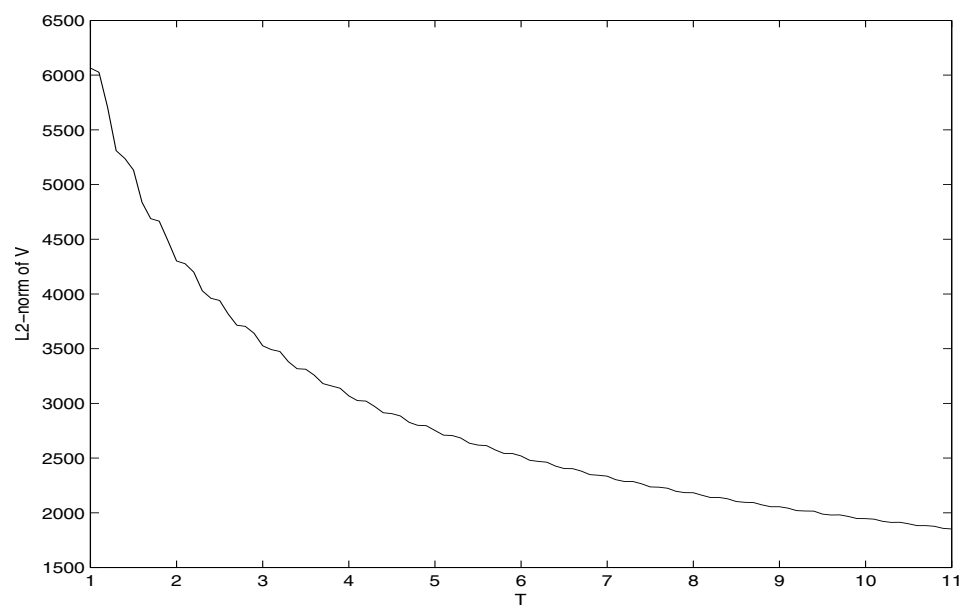

Figure $6 . L^{2}$-Norm of the control.

\section{Appendix A. Diophantine approximations}

Let us now prove the diophantine approximations we need to state our main results.

\section{A.1. Proof of Lemma 4.1}

In this section we prove Lemma 4.1. To do this let us rewrite the proof of [3], Lemma 1, page 121 (see also [14], Th. 4, p. 22) in our context and let us prove first the following result

Lemma A.1. For any fixed $\chi \in \mathbb{R}^{+}$, and for any $\epsilon>0$, the equation

$$
\left\|\alpha_{k} a-\chi\right\|_{1}<\frac{1}{k^{1+\epsilon}}
$$

has only a finite number of integer solutions $k>0$, for almost all $a \in(0,1)$. 
Proof of Lemma A.1. Set $e>0$ and select $K>1$ such that

$$
\sum_{k \geq K} \frac{1}{k^{1+\epsilon}} \leq e \text { and } \frac{\chi}{\alpha_{K}}<1
$$

For each $k \geq K$, consider the intervals of radius $\frac{1}{\alpha_{k} k^{1+\epsilon}}$ with centers

$$
\frac{0+\chi}{\alpha_{k}}, \frac{1+\chi}{\alpha_{k}}, \ldots, \frac{k_{0}-1+\chi}{\alpha_{k}}
$$

where $k_{0}=k_{0}(k)$ is the largest integer such that

$$
\frac{k_{0}-1+\chi}{\alpha_{k}}<1
$$

Consider the $a$ 's such that inequality (49) has infinitely many solutions. Thus, there exist $k \geq K$ and an integer $p=p(k)$ such that

$$
\left|a-\frac{p+\chi}{\alpha_{k}}\right|<\frac{1}{\alpha_{k} k^{1+\epsilon}} .
$$

As $a \in(0,1)$ then $p \leq k_{0}(k)$ and thus $a$ is in one of our intervals. The measure of the union of these intervals is bounded by

$$
\sum_{k \geq K} k_{0} \frac{2}{\alpha_{k} k^{1+\epsilon}}
$$

Thanks to (50) and (51), this bound is lower than

$$
4 e
$$

for every $e>0$. Thus the Lebesgue measure of the set of $a$ 's, such that the inequality (49) has infinitely many solutions, is zero. This concludes the proof of Lemma A.1.

Applying Lemma A.1 with $\chi=0$ and $\chi=\frac{1}{4}$, we get, for every $\epsilon>0$ and $i \in\{1,2\}$, the existence of $A_{i} \subset(0,1)$ of Lebesgue measure 1 such that, for any $a_{i} \in A_{i}$, we have

$$
\left\|\mid \alpha_{k} a_{i}\right\| \|_{i} \geq \frac{1}{k^{1+\epsilon}}
$$

for every $k$ in $\mathbb{N}$, except for a finite number of $k$ (this finite number of such $k$ depends on $a_{i}$ ). Therefore, for any $i \in\{1,2\}$ and for any $a_{i} \in A_{i}$, we can find $c_{i}>0$ such that we get Lemma 4.1 .

Remark A.2. To prove Lemma A.1, we need to know the first term of the Taylor expansion of the sequence $\left(\alpha_{k}\right)$. However to prove a controllability result in a larger set of initial conditions, we need to have a more precise estimation on the sequence $\left(\alpha_{k}\right)$. See Lemma 4.3 where the first three terms of the Taylor expansion of the sequence $\left(\alpha_{k}\right)$ are needed (see also Rem. A.5). 


\section{A.2. Proof of Lemma 4.3}

In this section we prove Lemma 4.3. To do this let us recall the following result:

Lemma A.3. [14], Th. 6, p. 22) There exists a nonempty uncountable subset $\hat{B}$ of $(0,1)$, with its Lebesgue measure null, such that, for every $b$ in $\hat{B}$, there exists $C_{8}>0$ such that, for any $k \in \mathbb{N}^{*}$, we have

$$
\|\mid k b\|_{1} \geq \frac{C_{8}}{k}
$$

We can deduce from Lemma A.3, the following result:

Lemma A.4. Let $\chi$ and $\chi^{\prime}$ be two non negative rational numbers. Then for every $b$ in $\hat{B}$, there exists $C_{9}>0$ such that, for every $k>0$, we have

$$
\left\|\mid\left(k+\chi^{\prime}\right) b+\chi\right\|_{1} \geq \frac{C_{9}}{k} .
$$

Proof of Lemma A.4. Let $(n, m)$ and $\left(n^{\prime}, m^{\prime}\right)$ be two couples of relative co-prime integers such that $\chi=\frac{n}{m}$ and $\chi^{\prime}=\frac{n^{\prime}}{m^{\prime}}$. Let $b \in B$. Due to Lemma A.3, we have for any $k>0$.

$$
\begin{aligned}
\left\|\left(k+\chi^{\prime}\right) b^{\prime}+\chi\right\| \|_{1} & =\left\|\mid\left(k+\frac{n^{\prime}}{m^{\prime}}\right) b+\frac{n}{m}\right\| \|_{1} \\
& =\left\|m\left(m^{\prime} k+n^{\prime}\right) b+n m^{\prime}\right\|_{1} \\
& \geq \frac{C_{8}}{m\left(m^{\prime} k+n^{\prime}\right)} \geq \frac{C_{9}}{k}
\end{aligned}
$$

where $C_{9}=C_{9}\left(n^{\prime}, m^{\prime}, m\right)$, and we get Lemma A.4.

Proof of Lemma 4.3. Let $B_{1}=\hat{B} \backslash\left\{b \in \hat{B}, \exists k \in \mathbb{N}^{*}, \alpha_{k} b \in \mathbb{N}\right\}$ and $B_{2}=\hat{B} \backslash\left\{b \in \hat{B}, \exists k \in \mathbb{N}^{*}, \alpha_{k} b+\frac{1}{4} \in \mathbb{N}\right\}$.

We first prove that $B_{1}$ and $B_{2}$ are nonempty.

For every $k \in \mathbb{N}^{*}, \alpha_{k}<k$. Thus, there exists at most $(2 k-1)$ elements $b$ in $\hat{B}$ such that $\alpha_{k} b \in \mathbb{N}$ and at most $2 k$ elements $b$ in $\hat{B}$ such that $\alpha_{k} b+1 / 4 \in \mathbb{N}$. Thus $\left\{b \in \hat{B}, \exists k \in \mathbb{N}^{*}, \alpha_{k} b \in \mathbb{N}\right\}$ and $\left\{b \in \hat{B}, \exists k \in \mathbb{N}^{*}, \alpha_{k} b+\frac{1}{4} \in \mathbb{N}\right\}$ are countable. As $\hat{B}$ is uncountable (see [14]), $B_{1}$ and $B_{2}$ are non empty and uncountable.

Let $i \in\{1,2\}$ and let $b \in B_{i}$. Thanks to Lemma 4.3, there exists $\bar{C}_{i}>0$, such that \|\|$\left(k-\frac{1}{2}\right) b\|\|_{i} \geq \frac{\bar{C}_{i}}{k}$. By (20), there exists $k_{i} \in \mathbb{N}^{*}$, such that for every $k \geq k_{i},\left|\alpha_{k}-\left(k-\frac{1}{2}\right)\right| \leq \frac{\bar{C}_{i}}{2 k}$. Thus, $\forall k \geq k_{i},\left.\left\|\left|\alpha_{k} b\right|\right\|\right|_{i} \geq \frac{\bar{C}_{i}}{2 k}$.

For $k<k_{i}$, let $D_{k}=k\left\|\alpha_{k} b\right\|_{i}$, and let $D=\min _{0<k<k_{i}} D_{k}$. By the construction of $B_{i}, D>0$. Let $c_{i}=\min \left(D, \frac{\bar{C}_{i}}{2}\right)$, then

This concludes the proof of Lemma 4.3.

$$
\forall k \in \mathbb{N}^{*},\left|\left\|\alpha_{k} b \mid\right\|_{i} \geq \frac{c_{i}}{k}\right.
$$

Remark A.5. Note that the key-point of the proof of Lemma A.4 is the rationality of the first term of the Taylor expansion of the sequence $\left(\alpha_{k}\right)$ and the nullity of the three following terms of the Taylor expansion. For an other sequence of eigenvalue $\left(\alpha_{k}\right)$, the equivalent diophantine approximations seems to be an open question.

Acknowledgements. The authors would like to thank M. Tucsnak for useful discussions.

\section{REFERENCES}

[1] C. Baiocchi, V. Komornik and P. Loreti, Ingham type theorems and applications to control theory. Boll. Unione Mat. Ital., Sez. B, Artic. Ric. Mat. 2 (1999) 33-63.

[2] V. Balamurugan and S. Narayanan, Shell finite element for smart piezoelectric composite plate/shell structures and its application to the study of active vibration control. Finite Elem. Anal. Des. 37 (2001) 713-738. 
[3] J.W.S. Cassels, An introduction to diophantine approximation. Moskau: Verlag 213 S. (1961).

[4] E. Crépeau, Exact boundary controllability of the Boussinesq equation on a bounded domain. Diff. Int. Equ. 16 (2003) 303-326.

[5] E. Crépeau, Contrôlabilité exacte d'équations dispersives issues de la mécanique. Thèse de l'Université de Paris-Sud, avalaible at www.math.ursq.fr/ ${ }^{\sim}$ crepeau/PUBLI/these1.html.

[6] P. Destuynder, I. Legrain, L. Castel and N. Richard, Theorical, numerical and experimental discussion of the use of piezoelectric devices for control-structure interaction. Eur. J. Mech., A/Solids 11 (1992) 97-106.

[7] P. Destuynder, A mathematical analysis of a smart-beam which is equipped with piezoelectric actuators. Control Cybern. $\mathbf{2 8}$ (1999) 503-530.

[8] M. Fliess, H. Mounier, P. Rouchon and J. Rudolph, Linear systems over Mikusinski operators and control of a flexible beam. ESAIM: Proc. 2 (1997) 183-193.

[9] P. Germain, Mecanique, Tome II. Ellipses (1996).

[10] D. Halim and S.O.R. Moheimani, Spatial Resonant Control of Flexible Structures - Applications to a Piezoelectric Laminate Beam. IEEE Trans. Control Syst. Tech. 9 (2001) 37-53.

[11] D. Halim and S.O.R. Moheimani, Spatial $\mathrm{H}_{2}$ control of a piezoelectric laminate beam: experimental implementation. IEEE Trans. Control Syst. Tech. 10 (2002) 533-546.

[12] W.S. Hwang and H.C. Park, Finite element modeling of piezoelectric sensors and actuator. AIAA Journal 31 (1993) $930-937$.

[13] A.E. Ingham, Some trigonometrical inequalities with applications to the theory of series. Math. Zeitschr. 41 (1936) 367-379.

[14] S. Lang, Introduction to diophantine approximations. Springer-Verlag (1991).

[15] S. Leleu, Amortissement actif des vibrations d'une structure flexible de type plaque à l'aide de transducteurs piézoélectriques. Thèse, ENS de Cachan (2002).

[16] J.L. Lions, Contrôlabilité exacte, perturbations et stabilisation de systèmes distribués, Tome 1, Contrôlabilité exacte, Collection de recherche en mathématiques appliquées 8 (Masson, Paris), 1988. distribués, Masson, Paris (1988).

[17] L. Meirovitch, Elements of vibration analysis, Düsseldorf, McGraw-Hill (1975).

[18] R. Rebarber, Spectral assignability for distribued parameter systems with unbounded scalar control. SIAM J. Control Optim. 27 (1989) 148-169.

[19] L. Rosier, Exact boundary controllability for the linear KdV equation - a numerical study. ESAIM: Proc. 4 (1998) $255-267$.

[20] J. Rudolph and F. Woittennek, Flatness based boundary control of piezoelectric benders. Automatisierungstechnik 50 (2002) $412-421$.

[21] R.S. Smith, C.C. Chu and J.L. Fanson, The design of $H_{\infty}$ controllers for an experimental non-collocated flexible structure Problem. IEEE Trans. Control Syst. Tech. 2 (1994) 101-109.

[22] S. Tliba and H. Abou-Kandil, $H_{\infty}$ controller design for active vibration damping of a smart flexible structure using piezoelectric transducers, in 4th Symp. IFAC on Robust Control Design (ROCOND 2003), Milan, Italy (2003).

[23] M. Tucsnak, Regularity and exact controllability for a beam with piezoelectric actuator. SIAM J. Control Optim. 34 (1996) 922-930. 